\title{
Inflammatory Cytokines Link Obesity and Breast Cancer
} Nalin Siriwardhana', Rett Layman², Ayub Karwandyar², Shiwani Patel2, Blair Tage ${ }^{2}$, Matthew Clark², Jessica Lampley², Courtney Rhody ${ }^{2}$,
Erica Smith², Arnold M Saxton', Naima Moustaid-Moussa ${ }^{1 *}$ and Jay Wimalasena ${ }^{2 *}$

${ }^{1}$ Department of Animal Science and Obesity Research Center, University of Tennessee, Knoxville, Tennessee 37996

${ }^{2}$ Department of Obstetrics and Gynecology, Graduate School of Medicine, University of Tennessee Medical Center, Knoxville, Tennessee 37920

\begin{abstract}
The risk of postmenopausal breast cancer is significantly increased by obesity. Further, low grade chronic inflammation, a hallmark of obesity, can contribute to detrimental health effects including high cancer incidence. Our goal is to understand the molecular basis for obesity-breast cancer interactions and dissect the role of inflammatory mediators secreted by adipocytes and breast cancer cells. Accordingly, we developed a three-dimensional (3D) coculture system to facilitate direct cell-cell interactions and also performed media transfer from adipocyte cultures to growing breast cell cultures. The co-culture system will facilitate both adipocyte and breast cell growth in an environment closely mimicking in-vivo tumor microenvironment. Co-cultures of human primary adipocytes obtained from lean and obese women with MCF10A, MCF7 and MDAMB231 breast cells (non-cancerous epithelial cells, cancerous and invasive breast cancer cells, respectively), led to cell type specific changes in secretion of several pro-inflammatory cytokines, such as IL-6 and TNFa, compared to monocultures. Regulation of cytokine secretion of breast cells by adipocytes and vice versa indicates the two-way communication between breast cells and adipocytes. Further, 3D co-culture and adipocyte conditioned media transfer experiments demonstrated that obese adipocytederived conditioned media can promote higher growth of breast cancer cells compared to that from lean adipocytes. Moreover, obese adipocyte conditioned media increased the activation of nuclear factor-KB (NF-KB) family members in breast cells, compared to lean adipocyte-derived media. Our results provide a novel model system to study adipocytebreast cancer cell interactions which may underlie the link between breast cancer and obesity and also demonstrate that inflammatory cytokines and other secreted factors are important in this interaction.
\end{abstract}

Keywords: Obesity; Breast cancer; Adipocytes; Inflammation; Cytokines

\section{Introduction}

Obesity has become a critical problem in the US and worldwide and results in a variety of chronic diseases including diabetes, cardiovascular disease and cancer [1]. Clinical evidence directly suggests that obesity has a significant impact on breast cancer progression and specifically that obesity in postmenopausal women doubles their risk for developing breast cancer and also acts as a risk factor for metastasis [2-5]. Recent clinical evidence also suggests that not only obesity but also other metabolic diseases such as the metabolic syndrome or type 2 diabetes can increase the risk of breast cancer. Additionally metformin, an AMPK activator, has been found to improve the survival of breast cancer in patients with type 2 diabetes [6,7]. These studies indicate the importance of metabolic/hormonal imbalances such as those observed in obesity in breast cancer. Of particular interest to us, very few studies have addressed the mechanisms by which obesity enhances breast cancer occurrence and progression. It has been proposed that adipocytederived estradiol may induce breast cancer $[2,8]$. Further, adipocytederived inflammatory cytokine profiles exhibit significant differences between lean and obese individuals and this unbalanced inflammatory profile (increased pro-inflammatory cytokine and decreased antiinflammatory cytokine secretion) in the obese condition may play a role in worsening both metabolic disorders and breast cancer progression [9-13]. Since the breast stroma is mostly constituted of adipocytes and their precursors, it is logical to propose that there are molecular interactions between breast cells and adipose tissue components which may underlie promotion of tumorigenesis. Chronic inflammation is associated with both obesity and breast cancer progression [8] and visceral adipose tissue is a major source of inflammatory mediators, and these may exert profound effects on breast cancer cells $[12,13]$. In this communication we have co-cultured human visceral adipocytes isolated from lean or obese women together with cancerous and noncancerous breast epithelial cells in three dimensional (3D) co-cultures to dissect molecular and cellular interactions between adipocyte and breast cancer cells. This system facilitates direct cell-cell interactions mimicking in-vivo conditions and allows investigation of the effects of adipocytes and their secretory cytokines on breast cell biology and vice versa. Our results demonstrate the two-way communication between the adipocytes and breast cancer cells in 3D co-cultures and further support the role for adipocyte-derived adipokines in modulating breast cancer cell growth and metabolism.

\section{Methods and Procedures}

Age-matched visceral preadipocytes from lean and obese women were obtained from Zenbio (Research Triangle Park, NC). The preadipocytes were cultured and differentiated in monolayer cultures according to supplier's procedures. After differentiation, cells were maintained in adipocyte maintenance media purchased from the supplier. Conditioned media ( 1 day old) obtained from differentiated mature adipocytes were mixed with standard MCF10A culture media at a 1:1 ratio and used for media transfer experiments. Prepared media mixtures were added into 1-day-old growing breast cell cultures seeded at the concentration of 5,000 cells / well of 96-well plates. This experimental setup was used to measure cell growth differences assayed

*Corresponding authors: Dr. Jay Wimalasena, University of Tennessee Medical Center, 1924 Alcoa Highway, Knoxville, TN, 37920 USA, Tel: 865-305-8961; Fax 865-305-6863; E-mail: jwimalas@utmck.edu

Dr. Naima Moustaid-Moussa, University of Tennessee Institute of Agriculture, 201L McCord Hall, 2640 Morgan Circle Drive, Knoxville, TN 37996, USA, Tel: 865-974-6255; Fax: 865-974-9043; E-mail: E-mail: moustaid@utk.edu

Received November 21, 2011; Accepted January 03, 2012; Published March 20, 2012

Citation: Siriwardhana N, Layman R, Karwandyar A, Patel S, Tage B, et al. (2012) Inflammatory Cytokines Link Obesity and Breast Cancer. J Metabolic Synd 1:102. doi:10.4172/2167-0943.1000102

Copyright: @ 2012 Siriwardhana N, et al. This is an open-access article distributed under the terms of the Creative Commons Attribution License, which permits unrestricted use, distribution, and reproduction in any medium, provided the original author and source are credited. 
by MTT assay and cell lysates prepared for TNF $\alpha$ and NF- $\kappa$ B assays. ELISA assays, including multiplex cytokine ELISAs, were performed according to manufacturer's guidelines.

Three dimensional (3D) cultures and 3D co-cultures were performed with additional modifications of previously described 3D breast epithelial culture methods [14,15]. Briefly, $400 \mu \mathrm{l}$ of Growth Factor-Reduced Matrigel Matrix (BD Biosciences, Bedford, MA) was added into each well of 24-well culture plates and placed in the cell culture incubator for $20 \mathrm{~min}$. Five thousand breast cells and/or 45,000 adipocytes were mixed with breast cell adipocyte culture media (MCF10A media and adipocyte culture media at a 1:1 ratio) and plated on top of the base layer of Matrigel. Cultures were maintained in $5 \% \mathrm{CO}_{2}$ at $37^{\circ} \mathrm{C}$. Media were carefully replaced every 2 days, and conditioned media were collected and stored at $-80^{\circ} \mathrm{C}$ for cytokine assays. Growing co-cultures were photographed every 3 days at our microscopic image facility and the diameters of acini-like spheroids were recorded. All experiments were conducted in triplicate and repeated two times to verify the results. Cell lysates in Figure 4B were prepared according to instructions of RayBiotech (Atlanta, GA) and Active Motif (Carlsbad, CA), respectively.

\section{Results and Discussion}

We co-cultured pre-adipocytes derived from either lean or obese women with MCF10A, MCF7, and MDAMB231 breast epithelial cells in $3 \mathrm{D}$ co-cultures in Matrigel. As shown in Figure 1A, MCF10A cells formed round, growth restricted spheroids, while MCF7 cells formed growth unrestricted irregular structures (Figure 1B). MDAMB231 cells formed mixed cellular masses with elongated extensions (Figure 1C). Figure 1D shows differentiated obese adipocytes grown in 3D culture with inter cellular connections. In the MCF10A, MCF7 and MDAMB231/adipocyte co-cultures, there appeared to be structural connections between the two cell types, as represented in Figure 1E. The diameters of these cellular structures in the co-cultures were measured microscopically. The diameter of MCF7 structures increased when co-cultured with obese adipocytes compared to co-cultures with lean adipocytes (Figure 2B). This increase, suggestive of a greater cell number, was not observed with MCF10A cells. Since MDAMB231 cells did not form compact structures, their diameters could not be ascertained. In conditioned media transfer experiments, we tested effects of lean and obese adipocyte-conditioned media on the growth of monolayers of breast cancer cells. When compared to lean derived adipocyte-conditioned media, obese adipocyte media induced timedependent increases in cell number in all three breast epithelial cell types (Figure 2A). The increases in cell number, although relatively modest, were statistically significant for MCF7 cells from 24-72 h following addition of conditioned media. Similar results were observed for MDAMB231cells at $72 \mathrm{~h}$. Studies have shown that obese adipocytederived adipokines and growth factors may regulate breast cell growth, as previously reviewed [16]. Thus, further studies are needed to identify specific factors derived from adipocytes which affect tumor growth and invasion.

It is well known that adipocytes secrete a variety of cytokines, and in the case of obese women, there is a significant increase in secretion of several pro-inflammatory adipokine compared to adipocytes from lean women $[9,10]$. Therefore, we measured levels of secreted inflammatory cytokines, which may regulate the cellular behavior of breast epithelial cells in the co-cultures. In our study, secretion of granulocyte colony stimulation factor (G-CSF) was higher in obese vs. lean monocultures (Figure 3). G-CSF secretion was greatly increased in cancer cells when compared to MCF10A cells. Co-culture changed G-CSF secretion in all

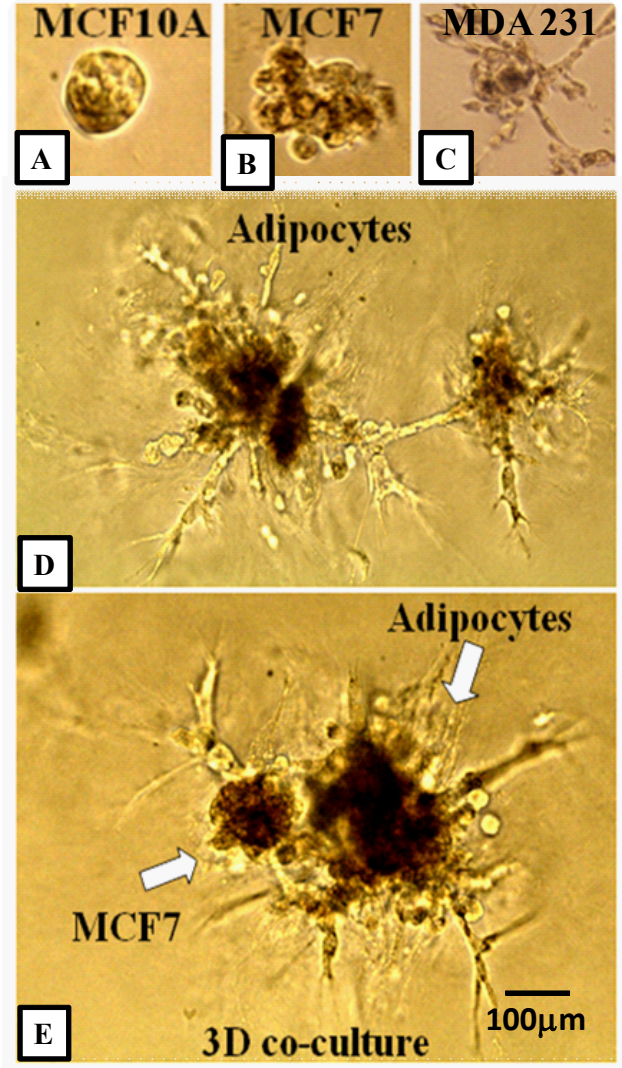

\begin{abstract}
Figure 1: Representative 3D cultures and co-cultures of differentiated adipocytes and breast cancer cells. Adipocytes from age-matched women were co-cultured with MCF10A, MCF7 and MDA 231 cells at a 9:1 ratio, respectively, in 3D Matrigel cultures. MCF10A cells formed size-restricted, regular round spheroids (A), and MCF7 cells formed size-unrestricted irregular spheroids (B), while invasive MDA 231 cells formed elongated cell clusters or interconnected cellular structures $(C)$, but not spheroids like MCF10A cells. D and E show connections between adipocytes and MCF7 cells. Arrows indicates the respective cell populations.
\end{abstract}

three breast cell lines. In MCF10A-lean adipocyte co-cultures, G-CSF secretion from lean adipocytes was increased several fold, compared to obese adipocytes. By contrast, in MDAMB231 cells, both lean and obese co-cultures suppressed G-CSF. In MCF7 co-cultures, G-CSF secretion from obese adipocytes was significantly increased compared to lean adipocytes. The order of G-CSF secretion in monoculture was MDAMB231 > MCF7 > MCF10A

IL-6 secretion (Figure 3B) was also up-regulated in MCF10A and MCF7 co-cultures with obese adipocytes, but was highly repressed in MDAMB231 co-cultures with lean or obese adipocytes, with a larger inhibitory effect seen with obese adipocytes. IL-6 secretion was 2-3fold higher in obese adipocytes compared to lean adipocytes, consistent with previous reports [17] and neither MCF10A nor MCF7 secreted detectable levels of IL-6 in 3D monocultures. The decrease in IL-6 in adipocytes and MDAMB231 co-cultures was over five-fold with obese adipocytes, while the reverse effects with less fold changes were observed with MCF10A and MCF7 cells.

IL-8 secretion from obese-derived adipocytes was significantly lower compared to lean adipocytes in 3D monocultures (Figure 3C). Furthermore, MCF10A cells did not secrete detectable IL-8; however, when co-cultured with adipocytes from lean or obese subjects, IL-8 
Citation: Siriwardhana N, Layman R, Karwandyar A, Patel S, Tage B, et al. (2012) Inflammatory Cytokines Link Obesity and Breast Cancer. J Metabolic Synd 1:102. doi:10.4172/2167-0943.1000102

Page 3 of 6

secretion was significantly reduced from adipocytes, and this reduction was more pronounced in co-cultures of MCF 10A with obese adipocytes, compared to adipocytes alone. This decrease is possibly due to MCF10A cells inhibiting secretion of IL- 8 by adipocytes. It could also result from uptake of IL-8 by MCF10A cells. MCF7 cells secreted low quantities of IL-8 and in co-cultures with lean adipocytes, there was a decrease in IL-8 compared to monocultures. However, in the presence of obese adipocytes, there was a significant increase in secreted IL-8, which suggests that MCF7 cells were stimulated to secrete IL- 8 by obese adipocytes or that IL- 8 secreted by obese adipocytes was stabilized by MCF7 cells, resulting in higher IL-8 in the medium. It is also possible that MCF7 cells stimulated obese adipocytes to secrete more IL-8. MDAMB231 cells secreted large quantities of IL8 , consistent with other reports on breast cancer cells [18]. IL-8 levels were significantly decreased when these cells were co-cultured with lean or obese adipocytes.

MCP-1 secretion by obese adipocytes was significantly higher than lean adipocytes (Figure 3D). This is in line with previous studies demonstrating higher MCP-1 production in obese vs. lean human subjects and from visceral vs. subcutaneous adipose tissue [19]. Breast cell lines secreted relatively lower MCP-1; in co-cultures with lean adipocytes and MCF10A or MCF7 cells, MCP-1 was relatively unchanged. In both MCF7 and MCF10A cell co-cultures with obese adipocytes, there was a large reduction in MCP-1 levels, suggesting that these two cell types may have decreased MCP-1 secretion by

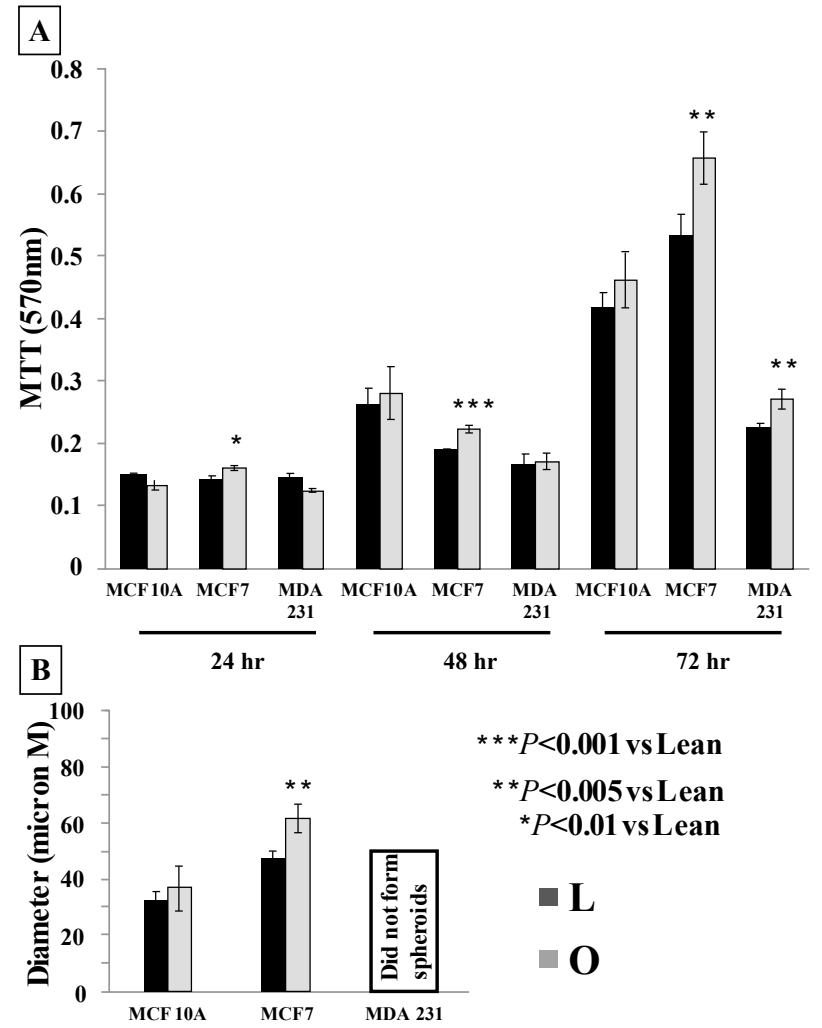

Figure 2: Effect of lean and obese adipocytes on breast cell growth. A. Lean and obese adipocytes from age-matched women were individually cultured, and the conditioned media was transferred to growing breast cell cultures. Cell growth was measured by MTT assay at 24, 48 and 72 h. B. Diameter of the structures shown in Figure 1 is shown (data are from triplicate cultures, mean +/- SE). L=Lean $\mathrm{O}=$ Obese

\begin{tabular}{|c|c|c|c|c|c|}
\hline Cytokine & Adipocyte & Media & MCF10A & MCF7 & MDAMB231 \\
\hline \multirow[t]{3}{*}{ Eotaxin } & Control & & 5.65 & 10.325 & 14.335 \\
\hline & Lean & 15.08 & 7.065 & 12.995 & 32.23 \\
\hline & Obese & 35.865 & 7.755 & 13.33 & 23.715 \\
\hline \multirow[t]{3}{*}{ GM-CSF } & Control & & 2.495 & 6.05 & 64.525 \\
\hline & Lean & 5.855 & 11.96 & 20.085 & 14.225 \\
\hline & Obese & 3.94 & 6.875 & 13.145 & 11.15 \\
\hline \multirow[t]{3}{*}{ IFN $\alpha 2$} & Control & & 5.945 & 8.98 & 11.53 \\
\hline & Lean & 26.605 & 15.565 & 19.26 & 9.485 \\
\hline & Obese & 13.05 & 12.04 & 14.555 & 9.485 \\
\hline \multirow[t]{3}{*}{$\operatorname{IL} 1 \alpha$} & Control & & 104.03 & 27.56 & 22.315 \\
\hline & Lean & 1.495 & 4.42 & 8.68 & 24.185 \\
\hline & Obese & 2.38 & 11.78 & 10.255 & 10.57 \\
\hline \multirow[t]{3}{*}{ IL-7 } & Control & & 2.94 & 4.59 & 27.855 \\
\hline & Lean & 10.45 & 7.33 & 8.735 & 9.79 \\
\hline & Obese & 8.735 & 7.335 & 10.14 & 8.035 \\
\hline \multirow[t]{3}{*}{ IP-10 } & Control & & 26.895 & 51.225 & 73.44 \\
\hline & Lean & 5.6 & 16.115 & 14.325 & 19.005 \\
\hline & Obese & 15.31 & 21.715 & 30.175 & 13.32 \\
\hline \multirow[t]{3}{*}{ MIP $1 \alpha$} & Control & & 4.77 & 4.78 & 7.045 \\
\hline & Lean & 4.945 & 4.955 & 6.36 & 7.705 \\
\hline & Obese & 3.88 & 6.36 & 5.31 & 3.7 \\
\hline
\end{tabular}

Table 1: Cytokines differentially expressed in 3D co-cultures of adipocytes and breast cells.

Breast cancer cells were either maintained in Matrigel as mono-cultures (contro designation), or co-cultured with adipocytes from lean or obese women. Cytokines secreted by monocultures of lean or obese adipocytes only, in Matrigel, are shown in the column labeled "Media". Values are in $\mathrm{pg} / \mathrm{ml}$. All the bolded values are significantly different compared to the lean adipocyte co-culture $(P<0.05)$.

obese adipocytes, or that the MCF7 or MCF10A cells bound and/or metabolized MCP-1 rendering it unavailable in the medium. In contrast to G-CSF, IL-6 and IL-8, MDAMB231 cells did not secrete detectable MCP-1 and these cells were much more efficient in inhibiting lean or obese adipocyte MCP-1 secretion and/or binding/metabolizing MCP1 than the other two cell lines. In this respect, co-culture of either lean or obese adipocytes with MDAMB231 cells showed a similar trend in secreted G-CSF, IL-6 and IL-8, in that co-culture with either lean or obese adipocytes significantly reduced secretion of these cytokines by MDAMB231 cells.

Of the twenty six cytokines measured in this study using 3D monoand co-culture media, 11 cytokines showed differential and significant changes (selected cytokines are shown in Figure 3 and others are shown in Table 1). Of the cytokines significantly changed, GM-CSF secretion was increased in monocultures in the order MCF10A < MCF7 < MDAMB231. In co-cultures with lean adipocytes, GM-CSF secretion from both MCF10A and MCF7 cells was increased. By contrast, GMCSF secretion was significantly reduced from MDAMB231 cells in cocultures with lean or obese adipocytes. Furthermore, compared to leanderived adipocytes, obese adipocytes decreased secretion of GM-CSF in co-cultures with all the breast cell lines. GM-CSF secretion by lean adipocytes was similar to that of obese adipocytes; however, compared to lean adipocytes, obese adipocytes were less stimulatory towards GMCSF secretion by MCF10A and MCF7 cells in co-cultures. Both lean and obese adipocytes in co-cultures with MDAMB231 cells drastically reduced the GM-CSF secretion by the latter.

Obese adipocytes secreted significantly more eotaxin (CCL11) than lean adipocytes in monoculture and Vasudevan et al. (2006) [20] reported that obesity promote the secretion of eotaxin. Similar to other cytokines/ interleukins, there was an increase in eotaxin secretion from MCF10A to MDMBA231 cells in monocultures. In lean 
adipocyte/MDAMB231 co-cultures, there was a significant $(\mathrm{P}<0.05)$ increase in eotaxin, suggesting a positive mutual interaction between the two cell types in secretion of eotaxin. On the other hand, in obese adipocyte/MDAMB231 cell co-cultures, there was a negative influence of the MDAMB31 cells on obese adipocyte secretion of eotaxin and/ or metabolism of eotaxin by MDAMB231 cells. Co-cultures did not change secretion of eotaxin by MCF10A and MCF7.

Secretion of IFNa2 also increased in monocultures (from MCF10A to MDAMB231 cells). Secretion of IFNa2 was higher in lean adipocyte than in obese adipocyte monocultures. In co-cultures with lean adipocytes, there were increased levels in MCF10A and MCF7 cells; the magnitude of changes was less in obese adipocyte co-cultures compared to lean adipocyte co-cultures. Interestingly, there was no effect of lean adipocytes or obese adipocytes on the MDAMB231 secretion of IFNa2 compared to highly significant effects of lean or obese adipocytes on secretion of other cytokines or interleukins from MDAMB231 cells. However, MDAMB231 cells decreased the quantity of IFNa2 secreted by adipocytes in monoculture, similar to effects of MCF10A and MCF7 cells on lean adipocytes IFNa2 secretion.

In contrast to all other cytokines, secretion of IL1 $\alpha$ was higher in MCF10A monocultures, compared to MCF7 and MDAMB231 cancer cells. In monoculture, lean and obese adipocytes secreted only small quantities of IL1a. However, both types of adipocytes drastically reduced secretion of this interleukin by MCF10A as well as MCF 7 . In MDAMBA231 co-cultures, only adipocytes from obese women reduced IL1a secretion.

IP-10 (CXCL10) secretion by monocultures of obese adipocytes was higher compared to lean adipocytes. However, both obese and lean adipocytes reduced IP-10 secretion from MCF10A and MDAMB231 cells. Adipocytes from lean subjects exerted a more pronounced decrease in IP-10 secretion from MCF10A and MCF7 cells, while obese adipocytes exerted larger inhibition in MDAMB231 cells. The secretion of IP-10 was in the order MDAMB231 > MCF7 > MCF10A in monocultures. In addition, in co-cultures of obese (but not lean) adipocytes with MDAMB231 cells, MIP 1 a secretion was significantly reduced.

Secretion of a variety of cytokines/interleukins is regulated by TNFa [11] and both adipocytes and breast cancer cells can secrete TNFa. The concentrations of TNFa in mono- and co-culture media are shown in Figure 4A,B. Obese adipocyte monocultures secreted more TNFa than lean adipocytes, as previously observed [21]. All the breast cell lines secreted TNFa, with MCF7 cells secreting the largest quantity in monoculture. In MCF10A cells co-cultured with lean adipocytes, the secretion of TNF $\alpha$ was similar compared to that by lean adipocytes alone. In MCF7 co-culture with lean adipocytes or obese adipocytes, there was a significant reduction in secreted $\mathrm{TNF} \alpha(\mathrm{P}<0.05)$. Interestingly, in all three cell types, obese adipocyte co-cultures accumulated significantly more TNFa than lean adipocyte co-cultures. Data in Figure 4B depicts TNFa levels in cell lysates in breast cells. Concentrations of TNFa in cell lysates were approximately ten-fold that of media in monocultures, and all three breast cell types had similar TNFa concentrations (normalized to total cell protein) in lysates. In co-cultures, both lean and obese adipocyte-conditioned media increased MCF10A and MCF7 cells cellular TNFa; lean adipocyte-conditioned medium induced significantly higher MCF10A TNFa than obese adipocyte-conditioned medium. Obese adipocyte-conditioned media significantly increased TNF $\alpha$ content in MDAMB231 and MCF7 cultures.

One of the well-known downstream targets of TNFa is the NF- $\kappa B$ pathway $[22,23]$. Using the nucleotide sequence to which all members

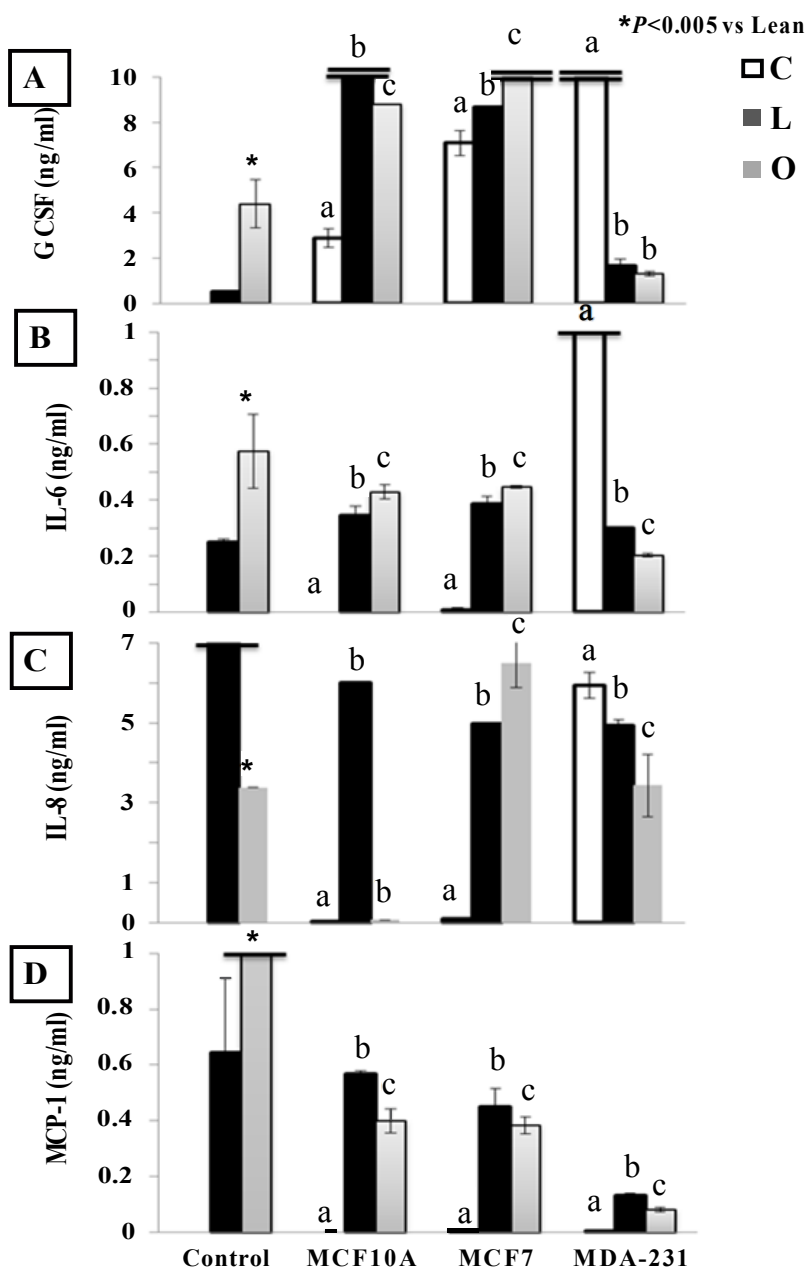

Figure 3: Cytokine changes in adipocyte and breast cell co-cultures. Media from 3D-co-cultures were assayed for secretion of selected cytokines (A-D). Data exceeding the upper detection limit were set to $10,001(\mathrm{pg} / \mathrm{ml})$ for statistical analysis. A double cross bar on the histogram indicates values were truncated at the upper detection limit. A single cross bar indicates values that exceed the depicted axis but did not exceed the upper detection limit. Different lower case letters indicate statistical difference, $p<0.05$. Control $=$ adipocyte mono-cultures, $\mathrm{C}=$ breast cell mono-cultures, $\mathrm{L}=\mathrm{Lean}, \mathrm{O}=\mathrm{Obese}$.

of the NF- $\kappa B$ family bind and specific antibodies against individual members of the NF- $\kappa B$ family, oligonucleotide-bound NF- $\kappa B$ was measured by a commercial ELISA kit. As shown in Figure 5, all five family members are present in all of the breast cell types. Using the same conditioned media transfer technique as in Figure 4B, we measured the effects of adipocyte-conditioned media on the activity of all five NF- $\mathrm{BB}$ family members. Conditioned media regulated activities in a cell-specific manner. The most prominent effects of obese vs. lean adipocyte media transfer were observed with MDAMD231 cells, where a significant increase in active p65, p50, p52, Rel B and Rel C was observed with obese adipocyte-conditioned media compared to lean adipocyte-conditioned media. In MCF7 cells, a similar pattern was observed for p65, p50, p52, and Rel C. However, the changes in MCF7 cells were relatively modest. In MCF10A cells, which had lower NF- $\kappa B$ family member concentrations compared to cancer cells except for Rel C, both obese and lean adipocyte-conditioned media exerted similar positive effects. Clearly, obese adipocyte-conditioned media had a larger stimulatory effect than lean adipocyte-conditioned media 


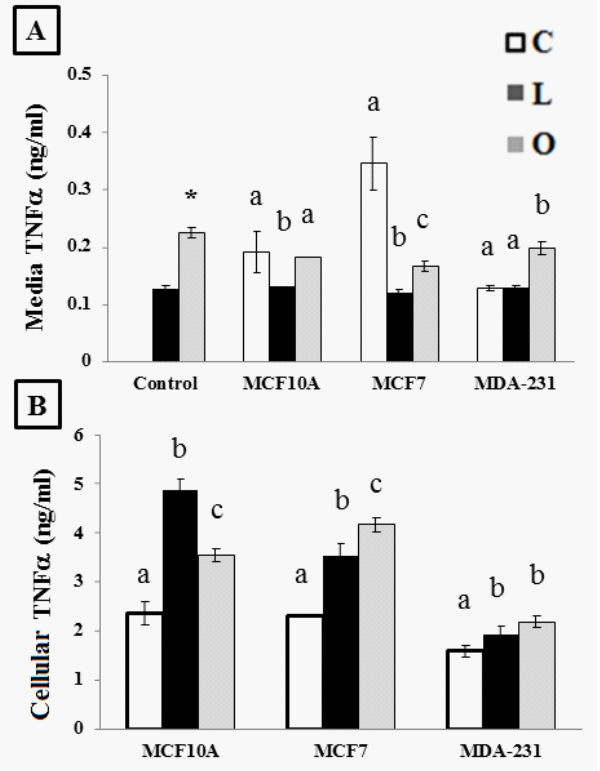

Figure 4:TNF $\alpha$ changes in co-culture media and adipocyte conditioned media transferred to breast cell cultures. Media and cell lysates were assayed for $\mathrm{TNF} \alpha(\mathrm{A} \& \mathrm{~B})$ following adipocyte conditioned media transfer to breast cell monocultures. Different lower case letters indicate statistical difference, ${ }^{*} p<0.05$. $C=$ breast cell mono-cultures, Control = adipocyte monocultures, $\mathrm{C}=$ breast cell mono-cultures, $\mathrm{L}=\mathrm{L}$ ean, $\mathrm{O}=$ Obese

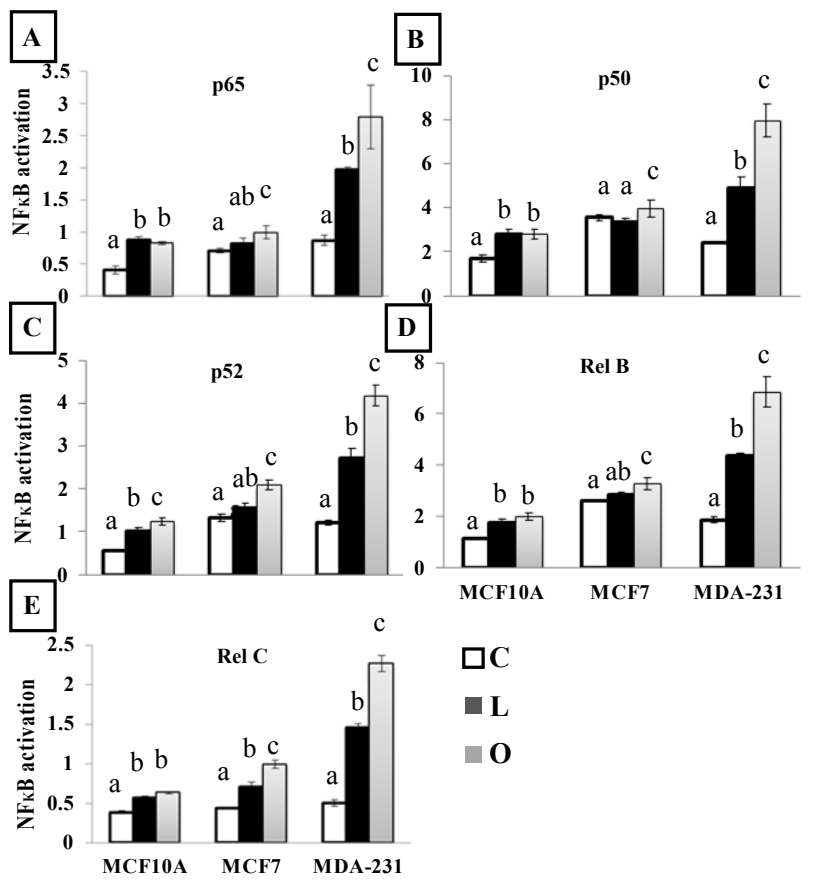

Figure 5: NF-kB activation changes in adipocyte conditioned media transferred to breast cell cultures. Adipocyte conditioned media-exposed breast cells were assayed for NFKB activation using an ELISA assay. Different lower case letters indicate statistical difference, $p<0.05$. $C=$ Breast cell monocultures, $\mathrm{L}=$ Lean, $\mathrm{O}=$ Obese.

on NF- $\kappa B$ family member activities in MDAMB231 cancer cells.

Our results demonstrate that co-culturing breast cell lines with adipocytes had significant effects on their growth. Further, obese adipocytes had a greater effect on breast cancer cells, especially on MCF7 cells. It is possible that cell-to-cell contact had a potential role here and/or that adipocyte secretions (such as cytokines) may have produced these effects. Our data suggests that the breast cell growth and metabolism is regulated by adipocyte-secreted cytokines. Although, we have not identified specific factors secreted by adipocytes which regulate breast cancer cell growth and NF- $\kappa B$ expression in these cells, the methods we have developed can be utilized to identify the molecules which mediate the two way communication between adipocytes and breast cells.

The effects of co-cultures on cytokine secretion are highly complex. We identified 11 cytokines that changed out of the twenty six measured. Lean and obese adipocytes exhibited substantial differences in secretion of many of these cytokines in 3D monocultures, except for GM-CSF, IL1a, IL-7 and MIP1a. In general, MDMBA231 cells produced greater amounts of cytokines (except ILla and MCP-1) than either cancerous MCF7 or non-cancerous MCF10A cells. There was an increase in secretion in the order MCF10A < MCF7 < MDAMB231 cells, which is also the order of the malignant nature in the three breast cell lines.

The interaction between lean or obese adipocytes and the breast cells are highly complex, with both lean and obese adipocytes decreasing secretion of several cytokines from MDAMB231 cells (except for eotaxin and IL1a). In contrast, co-cultures enhanced secretion of some other cytokines from MCF10A (e.g., G CSF) and MCF7 cells, with notable exceptions such as IL1- $\alpha$ and IP-I0. In some cases, such as with IL-8, MCF7 cells appeared to decrease IL- 8 secretion by lean and obese adipocytes. For obese adipocytes /MCF7 co-cultures, there was a significantly higher secretion of IL-8 than in either obese adipocytes or MCF7 cells alone. These data strongly suggest mutual interaction between adipocytes and breast cells in regulating these cytokines.

What is remarkable is that the highly malignant MDAMB231 cell line behaves differently in this interaction, compared to the less malignant MCF7 cells. In general, we observed a significant difference between lean and obese adipocyte co-cultures with breast cells (cell growth, secretion of the majority of cytokines, as well as activation of the NF- $\kappa \mathrm{B}$ family of transcription factors). Remarkably, there were significant increases in activation of all NF- $\kappa B$ family members in MDAMB231 cells after conditioned media transferred from obese adipocytes compared to the lean counterparts. These increases were even higher than when obese adipocyte conditioned media was transfered to MCF7 or MCF10A cultures.

$\mathrm{NF}-\kappa \mathrm{B}$ activation is known to modulate several different cellular processes in cancer cells leading to cell growth, as pharmacological inhibition of NF- $\mathrm{KB}$ in cell lines suppresses proliferation and leads to apoptosis $[24,25]$. In agreement with our data that the invasive MDAMB231cells showed higher NF- $\kappa B$ activation, Spiller et al. (2011) [24] showed that NF- $\kappa B$ is activated and required for medulloblastoma tumor growth. Further, it has been shown that anti-inflammatory treatments that suppresses NF- $\kappa \mathrm{B}$ activation are promising anti-cancer agents [26-28]. We observed that conditioned media derived from obese adipocytes significantly increased MDAMB 231 cell growth compared to the lean counterpart [Figure 2] and this is in agreement with Park et al. (2007) [29] where they showed that NF- $\mathrm{BB}$ activation in breast cancer cells promote bone metastasis and obesity increases metastasis in patients and our data suggest that increased NF- $\kappa B$ activation due to obese condition media transfer can promote MDAMB 231 growth.

Our novel studies on the co-culture of lean /obese adipocytes with breast epithelial cells in a 3D matrix demonstrates two-way 
direct communication between adipocytes and breast cells, as occurs naturally in the human breast. These interactions appear to be specific for each breast cell type (non-cancerous, cancerous and invasive) and lean vs. obese adipocytes. Therefore, our technique provides novel tools to elucidate the molecular interactions between adipocytes and breast cancer cells; these interactions may contribute to the increased incidence and poorer prognosis of breast cancer in post-menopausal, obese women. Furthermore, separation of breast cells from adipocytes and monoculture of the breast cells following co-culture may reveal molecular changes in breast cells that occurred during the co-culture. Finally, the system we developed would be highly suitable for testing effects of dietary interventions that may lessen the effects of fat cell inflammation on breast cancer cells and ultimately decrease the risk of breast cancer in obesity.

\section{Acknowledgement}

We acknowledge the grant support by University of Tennessee (UT) Obesity Research Center and UT Graduate School of Medicine. Further, we extend our thanks to Misty Bailey for technical editing of the manuscript. Authors 2-9 are undergraduate research trainees.

\section{References}

1. (1998) Clinical Guidelines on the Identification, Evaluation, and Treatment of Overweight and Obesity in Adults--The Evidence Report. National Institutes of Health. Obes Res 2: 51S-209S.

2. Roberts DL, Dive C, Renehan AG (2010) Biological Mechanisms Linking Obesity and Cancer Risk: New Perspectives. Annu Rev Med 61: 301-316.

3. Conroy SM, Maskarinec G, Wilkens LR, White KK, Henderson BE, et al. (2011) Obesity and breast cancer survival in ethnically diverse postmenopausal women: the Multiethnic Cohort Study. Breast Cancer Res Treat 129: 565-574.

4. Gu JW, Young E, Patterson SG, Makey KL, Wells J, et al. (2011) Postmenopausal obesity promotes tumor angiogenesis and breast cancer progression in mice. Cancer Biol Ther 11: 910-917.

5. Healy L, Ryan A, Rowley S, Boyle T, Connolly E, et al. (2010) Obesity Increases the Risk of Postmenopausal Breast Cancer and is Associated with More Advanced Stage at Presentation But no Impact on Survival. Breast J 16: 95-97.

6. Goodwin P, Ligibel J, Stambolic V (2009) Metformin in Breast Cancer: time for action. J Clin Oncol 27: 3271-3273.

7. Bosco J, Antonsen S, Sorensen H, Pedersen L, Lash T (2011) Metformin and Incident Breast Cancer among Diabetic Women: A Population-Based CaseControl Study in Denmark. Cancer Epidemiol Biomarkers Prev 20: 101-111.

8. Brow K, Simpson E (2010) Obesity and Breast Cancer: Progress to Understanding the Relationship. Cancer Res 70: 4-7.

9. Kalupahana NS, Claycombe K, Moustaid-Moussa N (2011) (n-3) Fatty Acids Alleviate Adipose Tissue Inflammation and Insulin Resistance: Mechanistic Insights. Adv Nutr 2: 304-316.

10. Ouchi N, Parker JL, Lugus JJ, Walsh K (2011) Adipokines in inflammation and metabolic disease. Nat Rev Immunol 11: 85-97.

11. Goldberg J, Schwertfeger K (2010) Proinflammatory Cytokines in Breas Cancer: Mechanisms of Action and Potential Targets for Therapeutics. Curr Drug Targets 11: 1133-1146.

12. Nicolini A, Carpi A, Rossi G (2006) Cytokines in breast cancer. Cytokine Growth Factor Rev 17: 325-337.

13. Lyon DE, McCain NL, Walter J, Schubert C (2008) Cytokine comparisons between women with breast cancer and women with a negative breast biopsy. Nurs Res 57: 51-58.

14. Song X, Siriwardhana N, Rathore K, Lin D, Wang HC (2010) Grape Seed Proanthocyanidin Suppression of Breast Cell Carcinogenesis Induced by Chronic Exposure to Combined 4-(Methylnitrosamino)-1-(3-Pyridyl)-1Butanone and Benzo[a]Pyrene. Mol Carcinog 49: 450-463.

15. Siriwardhana N, Choudhary S, Wang HC (2008) Precancerous model of human breast epithelial cells induced by NNK for prevention. Breast Cancer Res Treat 109:427-441.
16. Vona-Davis L, Rose D P (2007) Adipokines as endocrine, paracrine, and autocrine factors in breast cancer risk and progression. Endocr Relat Cance 14: 189-206

17. Yudkin JS, Kumari M, Humphries SE, Mohamed-Ali V (2000) Inflammation, obesity, stress and coronary heart disease: is interleukin-6 the link? Atherosclerosis 148: 209-214.

18. Freund A, Jolivel V, Durand S, Kersual N, Chalbos D, et al. (2004) Mechanism underlying differential expression of interleukin-8 in breast cancer cells. Oncogene 23: 6105-6114.

19. Bruun JM, Lihn AS, Pedersen SB, Richelsen B (2005) Monocyte chemoattractant protein-1 release is higher in visceral than subcutaneous human adipose tissue (AT): implication of macrophages resident in the AT. J Clin Endocrinol Metab 90: 2282-2289

20. Vasudevan AR, Wu H, Xydakis AM, Jones PH, Smith EO, et al. (2006) Eotaxin and obesity. J Clin Endocrinol Metab 91: 256-261.

21. Cottam D, Fisher B, Ziemba A, Atkinson J, Grace B, et al. (2010) Tumor growth factor expression in obesity and changes in expression with weight loss: another cause of increased virulence and incidence of cancer in obesity. Surg Obes Relat Dis 6: 538-541.

22. Van Laere SJ, Van der Auwera I, Van den Eynden GG, Elst HJ, Weyler J, et al. (2006) Nuclear factor-kappa B signature of inflammatory breast cancer by cDNA microarray validated by quantitative real-time reverse transcription-PCR immunohistochemistry, and nuclear factor-kappa B DNA-binding. Clin Cancer Res 12: 3249-3256.

23. Vanden Berghe W, Vermeulen L, De Wilde G, De Bosscher K, Boone E, et al. (2000) Signal transduction by tumor necrosis factor and gene regulation of the inflammatory cytokine interleukin-6. Biochem Pharmacol 60: 1185-1195.

24. Spiller SE, Logsdon NJ, Deckard LA, Sontheimer H (2011) Inhibition of nuclea factor kappa-B signaling reduces growth in medulloblastoma in vivo. BMC Cancer 11: 136.

25. Demchenko YN, Kuehl WM (2010) A critical role for the NFאB pathway in multiple myeloma. Oncotarget 1: 59-68.

26. Peralta EA, Murphy LL, Minnis J, Louis S, Dunnington GL (2009) American Ginseng inhibits induced COX-2 and NFKB activation in breast cancer cells. J Surg Res 157: 261-267.

27. Spencer L, Mann C, Metcalfe M, Webb M, Pollard C, et al. (2009) The effect of omega-3 FAs on tumour angiogenesis and their therapeutic potential. Eur $J$ Cancer 45: 2077-2086

28. Antoon JW, White MD, Slaughter EM, Driver JL, Khalili HS, et al. (2011) Targeting NFKB mediated breast cancer chemoresistance through selective inhibition of sphingosine kinase-2. Cancer Biol Ther 11: 678-689.

29. Park BK, Zhang H, Zeng Q, Dai J, Keller ET, et al. (2007) NF-kappaB in breast cancer cells promotes osteolytic bone metastasis by inducing osteoclastogenesis via GM-CSF. Nat Med 13: 62-69. 